\title{
THE ROSAT OBSERVATIONS OF CLASSICAL NOVAE
}

\author{
M. ORIO ${ }^{1,2}$, H. ÖGELMAN ${ }^{1}$, S. BALMAN ${ }^{1}$ \\ 1. Department of Physics, $U$ Wisconsin, Madison, USA \\ 2. Osservatorio Astronomico di Torino, Italy
}

We observed a number of classical and recurrent novae in the Galaxy and the LMC with the ROSAT X-ray telescope and searched the archival data for other serendipitous observations. Preliminary results show that only 9 out of 37 observed objects were bright enough in X-rays to be detected with ROSAT, either in outburst or at quiescence.

Three basic mechanisms can cause X-ray emission from classical or recurrent novae. The first is hot hydrogen burning in a thin shell of the remnant envelope left on the white dwarf after the nova explosion. Hydrogen burning post-novae should be blackbody like emitters at nearly Eddington luminosity (as per the 'supersoft' X-ray sources). In our sample, only GQ Mus (Nova Mus 1983, see Ögelman et al. 1993; Shanley et al. 1995) and V1974 Cyg 1992 (Krautter et al. 1996) had these characteristics. Remarkably, among $10 \mathrm{LMC}$ novae that had an outburst in the last $47 \mathrm{yr}$ none was detected as a 'supersoft' X-ray source. The $3 \sigma$ upper limits for the blackbody temperatures of the post-nova white dwarfs are mainly in the range $20 \ldots 30 \mathrm{eV}$. A post-nova can also emit X-rays because of shocks occurring in the ejected shell (e.g. O'Brien et al. 1994). Three out of four classical novae that were observed in outburst displayed a hard X-ray component in the ROSAT energy band, which might be due to a shocked shell. Finally, $\mathrm{X}$-ray emission is expected from quiescent nearby novae because of accretion. Only four nearby accreting sources were detected; the ROSAT upper limits for the non-detected quiescent novae are $L_{\mathbf{x}}<10^{31} \ldots 10^{32} \mathrm{erg} \mathrm{s}^{-1}$, assuming a thermal plasma at $k T=\mathrm{a}$ few $\mathrm{keV}$.

\section{References}

Krautter, J., Ögelman, H., Starrfield, S., et al., 1996, Ap. J., 456, 788

O'Brien, T. J., Lloyd, H. M., Bode, M. F., 1994, MNRAS, 271, 155

Ögelman, H., Orio, M., Krautter, J., Starrfield, S., 1993, Nature, 361, 331

Shanley, L. Ögelman, H., Gallagher, J., et al., 1995, Ap. J., 438, L95 